\title{
CONSUMO DE ÁGUA PELA CULTURA DO CRISÂNTEMO ENVASADO SOB CONDIÇÕES DE CASA DE VEGETAÇÃO
}

\author{
Raquel Aparecida Furlan ${ }^{1}$, Tarlei Arriel Botrel $^{2}$ e Vital Pedro da Silva Paz $^{3}$
}

\begin{abstract}
RESUMO
O principal objetivo desta pesquisa foi avaliar o consumo de água pelo crisântemo (Dendrantema grandiflora cv. Puritan) cultivado em vasos sob condições de casa de vegetação. Buscando-se a melhor representatividade dos dados, o experimento foi conduzido na Granja Triângulo, localizada no município de Holambra, São Paulo, Brasil, empregando-se as práticas culturais tradicionais. O requerimento de água da cultura foi estimado comparando-se o peso dos vasos, durante cinco diferentes estágios de desenvolvimento da cultura, com a evaporação de um tanque classe A modificado (tanque reduzido) e de um atmômetro modificado, com a área foliar da planta. Em cada estágio (tratamento) foram avaliadas cinco plantas, resultando em cinco repetições por tratamento. Para maior abrangência das condições climáticas, foram realizadas medições durante uma semana, de agosto a dezembro de 1995 e de fevereiro a junho de 1996, num intervalo de coleta de dados de dois meses. Através de uma análise múltipla de regressão, os resultados mostraram que existe correlação estatística entre dados medidos e estimados e que o consumo de água pelo crisântemo pode ser estimado considerando-se a área foliar em cada estágio de desenvolvimento da cultura.
\end{abstract}

Palavras-chave: Dendrantema grandiflora, evapotranspiração, atmômetro, tanque reduzido, estufa

\section{WATER CONSUMPTION BY POTTED CHRYSANTHEMUM UNDER GREENHOUSE CONDITIONS}

\begin{abstract}
The main objective of this research was to evaluate the evapotranspiration rate of the chrysanthemum (Dendrantema grandiflora cv. Puritan), cultivated in pots under greenhouse conditions. In order to get best representative data, the experiment was conducted at the Triângulo farm located in Holambra municipality in São Paulo State of Brazil, employing traditional tillage practices. The water requirement of this crop was estimated by comparing the chrysanthemum pot weight, during five different development stages of crop, with the evaporation from a modified class A pan and from a modified atmometer, and with the crop leaf area index. In each stage (treatment) five plants were evaluated, resulting in five repetitions per treatment. For a more representative weather conditions, the measurements were performed from August to December 1995 and from February to
\end{abstract}

\footnotetext{
${ }^{1}$ M.Sc. Doutoranda em Irrigação e Drenagem, DER/ESALQ-USP, Av. Pádua Dias 11. CP. 09. 13418-900. Piracicaba, SP. Fone (019) 429-4217. e-mail: rafurlan@ carpa.ciagri.usp.br

2 Professor Associado, DER/ESALQ-USP

${ }^{3}$ Dr. Irrigação e Drenagem, DER/ESALQ-USP, Bolsista da FAPESP
} 
June 1996, on a two-month interval basis. A multiple regression analysis of the results showed that there is a statistical correlation between measured and estimated data, and also that the chrysanthemum evapotranspiration rate can be estimated considering the leaf area in each development stage of crop.

Key words: Dendrantema grandiflora, evapotranspiration rate, atmometer, modified class A pan

\section{INTRODUÇÃO}

A floricultura, no aspecto cultural e ecológico, salienta não só o uso de flores para ornamentação de cerimônias cívicas, civis ou religiosas mas, também, a preservação e divulgação de espécies nativas em extinção no seu habitat (Kampf, 1989).

Dentre as flores cultivadas em vasos, destaca-se o crisântemo (Dendranthema grandiflora Tzvelev.) planta ornamental comercializada em todo o mundo, pela beleza de sua inflorescência. As variedades de crisântemo apresentam porcentagem expressiva na demanda mundial de flores de corte e envasadas, ocupando cerca de $12 \%$ do mercado holandês de flores cortadas e 34\% do mesmo mercado para flores envasadas (Kampf et al., 1990). Em 1987 esta planta ornamental já se encontrava entre as flores com o maior volume das exportações brasileiras dirigido, principalmente, ao mercado europeu (Mercado das Flores, 1987).

Por se tratar de uma cultura de grande valor comercial, com alta tecnologia de produção e exigente em fotoperiodismo, sua produção é conduzida, geralmente, em ambientes protegidos, de maneira a fornecer flores ao mercado o ano todo.

O consumo de água pelo crisântemo e das plantas ornamentais é, no geral, pouco estudado, sendo a literatura a respeito praticamente inexistente. Deste modo, nota-se que há certa dificuldade por parte dos produtores em se fazer o manejo racional da irrigação nessas culturas, principalmente naquelas conduzidas em ambiente protegido, visto que, por apresentarem condições ambientais próprias, impedem o uso direto dos métodos já consagrados para a determinação da evapotranspiração. Certos autores, como Montero et al. (1985) e Rosenberg et al. (1989) afirmam que a evapotranspiração no interior das estufas corresponde a $60 \%$ e $80 \%$ da verificada anteriormente.

O presente trabalho teve por objetivo determinar o consumo de água pelo crisântemo, cultivado em vasos mantidos em ambiente protegido, através da comparação de seus pesos, em diferentes fases do desenvolvimento da planta com a evaporação de um atmômetro com escala de leitura ampliada e a evaporação do tanque reduzido, relacionandoas com a área foliar da planta, no estágio de desenvolvimento considerado.

\section{MATERIAL E MÉTODOS}

O experimento foi realizado de modo a não alterar as formas de plantio e condução da cultura, que seguiram a rotina de produção comercial das plantas de crisântemo cultivadas em vasos.
O experimento foi realizado na propriedade denominada Granja Triângulo, no município de Holambra, SP, cujas coordenadas geográficas são: latitude de $22^{\circ}, 33^{\prime}$ sul e longitude $47^{\circ}, 01$ ' oeste e $686 \mathrm{~m}$ de altitude.

A casa de vegetação onde foi instalado o experimento está disposta no sentido norte-sul, tem área de $4300 \mathrm{~m}^{2}, 5,0 \mathrm{~m}$ de pé direito e cobertura plástica de polietileno transparente de baixa densidade, com espessura de 100 micra, suportada por estrutura de ferro galvanizado (estufa pré-fabricada).

Foram utilizados vasos cônicos de barro (número 15) com volume aproximado de $1 l$, plantados com crisântemo (cv. Puritan). Coletaram-se dados de plantas com cinco idades diferentes, a cada duas semanas (estágios). Adicionou-se um volume conhecido de água a 5 vasos de cada estágio ( 5 repetições); o volume drenado de cada vaso também foi medido e, por diferença de pesagem, obteve-se o consumo de água. Procurando-se obter informações sobre o consumo de água pelo crisântemo cultivado sob diferentes condições climáticas, as avaliações foram repetidas em seis diferentes épocas, com intervalo de 2 meses entre elas.

Em todas as épocas de amostragem foram avaliadas as áreas foliares da cultura em cada estágio de desenvolvimento, através da comparação de peso seco de círculos de folhas frescas de área conhecida e o peso seco de todas as folhas das plantas contidas no vaso. Mediu-se a evaporação de dois atmômetros de Althenhofen (Althenhofen, 1985) com escala de leitura ampliada e em um tanque classe A, com diâmetro reduzido $(60 \mathrm{~cm}$ de diâmetro e $25 \mathrm{~cm}$ de altura).

$\mathrm{Na}$ análise do ajuste foram considerados o coeficiente de determinação $\left(R^{2}\right)$ e o índice de concordância (D) o qual reflete o grau a que os desvios observados em relação à média correspondem em magnitude e sinal aos desvios previstos em relação à média. Não se trata de uma medida de correlação ou associação no sentido formal, mas uma medida do grau a partir do qual as revisões dos modelos estão livres de erros. O teste D é uma medida padronizada, a fim de ser facilmente interpretada e possibilitar comparações cruzadas de suas magnitudes para uma variedade de modelos, independente de unidades (Willmott, 1981).

\section{Área foliar em função dos dias após o plantio das mudas}

Para se estudar o comportamento do aumento da área foliar em função da idade da planta, coletaram-se amostras de plantas em cada um dos cinco estágios de desenvolvimento considerados e determinou-se a área foliar (AF). Ajustou-se, por meio de análise de regressão, a área foliar (AF) em função de dias após o plantio das mudas (DAP). A equação foi ajustada aos dados coletados em cada época do ano, considerando-se também todas as épocas simultaneamente, de acordo com o modelo polinomial raiz quadrada a seguir: 


$$
\mathbf{A F}=\mathbf{a}+\mathbf{b} \sqrt{\mathrm{DAP}}+\mathbf{c} . \mathrm{DAP}
$$

sendo:

AF - área foliar, $\mathrm{m}^{2}$

DAP - dias após o plantio das mudas

a, b, c - coeficientes de regressão.

\section{Consumo de água pelo crisântemo}

As equações ajustadas para o consumo de água do crisântemo em função da evaporação do tanque reduzido e da área foliar, são válidas para o intervalo de 27 a 96 dias após o plantio das mudas (DAP) e seguem o modelo descrito a seguir:

$$
\mathbf{C A}=\mathbf{a}+\mathbf{b} \cdot \mathbf{A F}+\mathbf{c} . \mathbf{T R}
$$

sendo:

CA - consumo de água pelo crisântemo, mm

$\mathrm{AF}$ - área foliar, $\mathrm{m}^{2}$

TR - evaporação do tanque reduzido, $\mathrm{mm}$

a, b, c - coeficientes de regressão.

Para se correlacionar o consumo de água pela cultura do crisântemo, em função da AF e da leitura da evaporação do atmômetro de escala ampliada (ATM) utilizou-se o modelo descrito a seguir:

$$
\mathbf{C A}=\mathbf{a}+\mathbf{b} \cdot \mathbf{A F}+\mathbf{c} \cdot \mathbf{A T M}
$$

sendo:

ATM - evaporação do atmômetro de escala ampliada, mm a, b, c - coeficientes de regressão.

\section{RESULTADOS E DISCUSSÃO}

A Tabela 1 apresenta os parâmetros do ajuste da AF, o número de observações $(\mathrm{N})$, os coeficientes de determinação $\left(\mathrm{R}^{2}\right)$ e o índice de concordância (D), para cada período de coleta de dados e para o ano todo.

Tabela 1. Épocas, número de observações $(\mathrm{N})$, coeficientes de regressão ( $a, b$ e c $)$, coeficientes de determinação $\left(R^{2}\right)$ e índices de concordância (D) das equações da área foliar, em função dos dias após o plantio das mudas

\begin{tabular}{ccccccc}
\hline & & \multicolumn{5}{c}{ Coeficientes de regressão } \\
\cline { 3 - 5 } Época & $\mathrm{N}$ & $\mathrm{a}$ & $\mathrm{b}$ & $\mathrm{c}$ & $\mathrm{R}^{2}$ & $\mathrm{D}$ \\
\hline Ago/95 & 05 & $-1,5363$ & $-0,02347$ & 0,4138 & $0,8985^{*}$ & 0,869 \\
Out/95 & 05 & $-0,86212$ & $-0,01205$ & 0,2416 & $0,9830^{* *}$ & 0,996 \\
Dez/95 & 05 & $-1,52262$ & $-0,02528$ & 0,4372 & $0,9433^{* *}$ & 0,987 \\
Fev/96 & 06 & $-1,51018$ & $-0,01968$ & 0,3914 & $0,9797^{* *}$ & 0,995 \\
Abr/96 & 05 & $-1,44381$ & $-0,02387$ & 0,4148 & $0,9827 * *$ & 0,996 \\
Jun/96 & 05 & $-0,94388$ & $-0,11324$ & 0,2510 & $0,9541 * *$ & 0,990 \\
Ano todo & 31 & $-1,033632$ & 0,28325 & $-0,0141$ & $0,8794 * *$ & 0,968 \\
\hline
\end{tabular}

* Significativo a 0,05 de probabilidade pelo teste $\mathrm{t}$

** Significativo a 0,01 de probabilidade pelo teste $\mathrm{t}$

A Figura 1 mostra a curva da equação geral obtida e os dados medidos para a área foliar, em função dos dias após o plantio das mudas, para todo o período do experimento.

$\mathrm{O}$ índice D confirmou haver boa concordância dos dados estimados da variável AF, pela equação de regressão 1 , conforme pode ser observado na Figura 2; desta forma, pode-se estimar a área foliar das plantas de crisântemo, através da equação de regressão que utiliza dados de todos os períodos de coleta, sem que ocorram erros significativos.

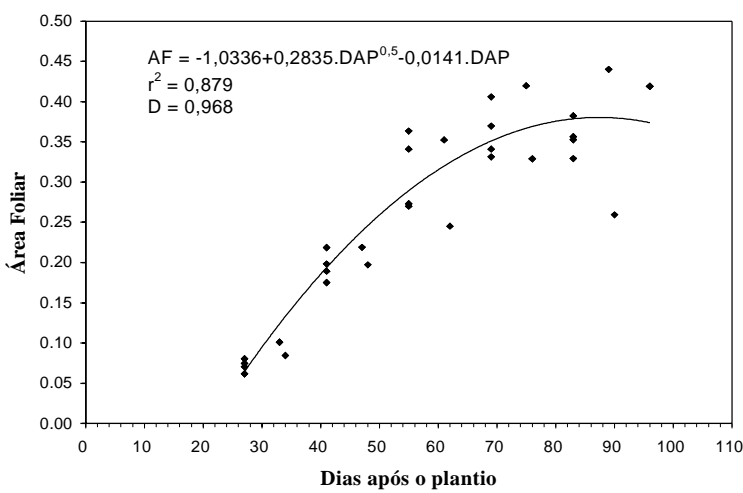

Figura 1. Correlação entre área foliar e dias após o plantio das mudas

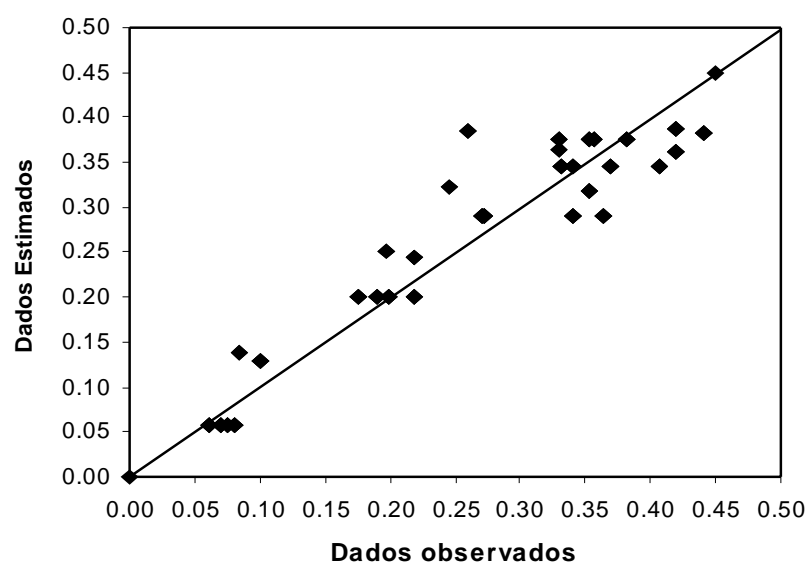

Figura 2. Dados medidos versus dados estimados pela equação polinomial raiz quadrada da área foliar, em função dos dias após o plantio das mudas

A Equação 2 foi ajustada por análise de regressão envolvendo o consumo de água pela cultura do crisântemo (CA) em função da AF e da TR. O ajuste foi feito considerando-se cada época de coleta e uma única equação envolvendo todos os dados observados durante o experimento. Utilizando-se todos os dados obtidos, obteve-se um índice D de 0,8828 e um coeficiente de correlação $\left(\mathrm{R}^{2}\right)$ de 0,6390 , sendo este significativo a 0,01 de probabilidade (Tabela 2 ).

Considerando-se individualmente cada período de coleta, os ajustes dos dados à Equação 2 foram melhores que aquele que envolve todo o período, o que se deve à maior homogeneidade dos dados climáticos.

Tabela 2. Épocas, número de observações $(\mathrm{N})$, coeficientes de regressão $(\mathrm{a}, \mathrm{b}$ e c $)$, coeficientes de determinação $\left(\mathrm{R}^{2}\right)$ e índice de concordância (D) das equações do consumo de água pelo crisântemo, em função da área foliar e da evaporação do tanque reduzido

\begin{tabular}{ccrcccc}
\hline Epoca & $\mathrm{N}$ & \multicolumn{4}{c}{ Coeficientes de regressão } & \\
\cline { 3 - 5 } & & $\mathrm{a}$ & $\mathrm{b}$ & $\mathrm{c}$ & $\mathrm{R}^{2}$ & $\mathrm{D}$ \\
\hline Ago/95 & 10 & 5,5787 & 28,0836 & 3,7786 & $0,8951^{* *}$ & 0,978 \\
Out/95 & 15 & $-11,0534$ & 28,4313 & 8,6351 & $0,9429^{* *}$ & 0,987 \\
Dez/95 & 15 & $-49,6806$ & 21,8358 & 29,226 & $0,8241^{* *}$ & 0,958 \\
Fev/96 & 18 & 8,6078 & 31,2712 & 1,7591 & $0,7067^{* *}$ & 0,921 \\
Abr/96 & 15 & $-0,2238$ & 24,3861 & 3,8101 & $0,9094^{* *}$ & 0,979 \\
Jun/96 & 15 & 0,8118 & 31,3255 & 2,7676 & $0,9106^{* *}$ & 0,979 \\
Ano todo & 88 & $-2,1062$ & 27,9109 & 5,19759 & $0,6390^{* *}$ & 0,883 \\
\hline
\end{tabular}

** Significativo a 0,01 de probabilidade pelo teste $\mathrm{t}$ 
A Equação 3, utilizando todos os dados obtidos, apresentou um índice D de 0,8773 e coeficiente de determinação $\left(R^{2}\right)$ de 0,7400 , este significativo a 0,01 de probabilidade, conforme a Tabela 3 na qual se encontram os parâmetros de ajuste do consumo do crisântemo (CA), o número de dados observados $(\mathrm{N})$, os coeficientes de determinação $\left(\mathrm{R}^{2}\right)$ e o índice $\mathrm{D}$.

Tabela 3. Épocas, número de observações $(\mathrm{N})$, coeficientes de regressão (a, b e c), coeficientes de determinação $\left(R^{2}\right)$ e índice de concordância (D) das equações do consumo de água pelo crisântemo, em função da área foliar e da evaporação do atmômetro de escala ampliada

\begin{tabular}{|c|c|c|c|c|c|c|}
\hline \multirow[t]{2}{*}{ Época } & \multirow[t]{2}{*}{$\mathrm{N}$} & \multicolumn{3}{|c|}{ Coeficientes de regressão } & \multirow[b]{2}{*}{$\mathrm{R}^{2}$} & \multirow[b]{2}{*}{$\mathrm{D}$} \\
\hline & & $\mathrm{a}$ & $\mathrm{b}$ & $\mathrm{c}$ & & \\
\hline Ago/95 & 10 & 57,006 & 28,0836 & $-22,6719$ & $0,8951 * *$ & 0,978 \\
\hline Out/95 & 15 & $-4,4860$ & 28,4313 & 7,1294 & $0,6757 * *$ & 0,721 \\
\hline Dez/95 & 15 & $-2,3088$ & 21,8358 & 6,4795 & $0,8970 * *$ & 0,976 \\
\hline Fev/96 & 18 & 3,9748 & 31,2712 & 3,9313 & $0,7818 * *$ & 0,944 \\
\hline $\mathrm{Abr} / 96$ & 15 & $-26,1359$ & 24,3861 & 18,6541 & $0,9245^{* *}$ & 0,983 \\
\hline Jun/96 & 15 & 8,4251 & 31,3255 & $-0,2178$ & $0,8872 * *$ & 0,974 \\
\hline Ano todo & 88 & $-4,2292$ & 29,2376 & 0,99944 & $0,7400^{* *}$ & 0,877 \\
\hline
\end{tabular}

** Significativo a 0,01 de probabilidade pelo teste $\mathrm{t}$

Considerando-se individualmente cada período de coleta, os ajustes dos dados das Equações 2 e 3 foram melhores que aquele que envolve todo o período, o que se deve à maior homogeneidade dos dados climáticos.

As Figuras 3a e 3b mostram a dispersão dos dados medidos em relação aos dados estimados, considerando-se todos os dados obtidos durante o experimento.

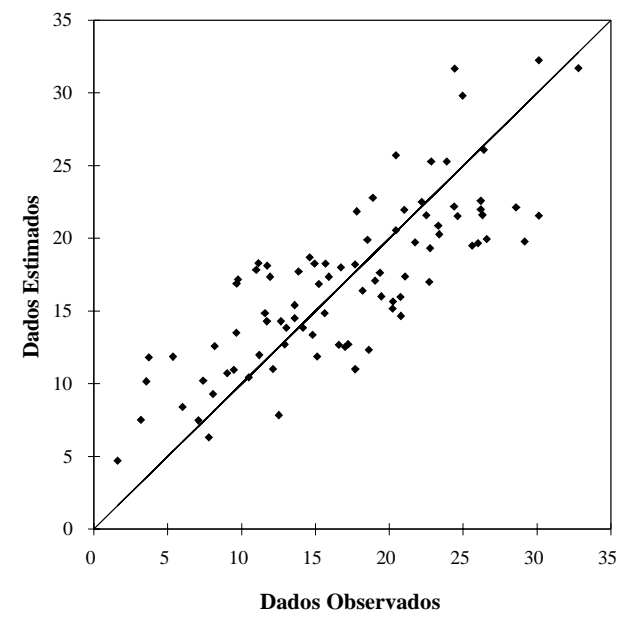

Figura 3a. Dados medidos do consumo de água pelo crisântemo versus dados estimados a partir da área foliar e da evaporação do tanque reduzido

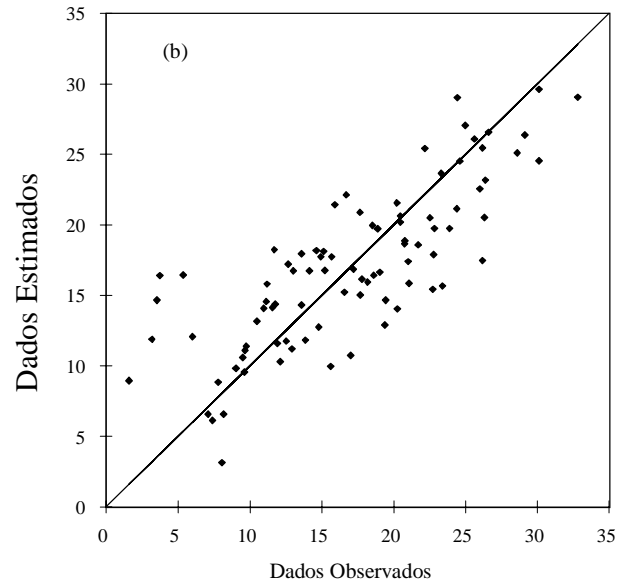

Figura 3b - Dados medidos do consumo de água pelo crisântemo versus dados estimados a partir da área foliar e da evaporação do atmômetro de escala ampliada, envolvendo todos os dados coletados

\section{CONCLUSÕES}

De acordo com os resultados obtidos, concluiu-se que:

1. O consumo de água pelo crisântemo pode ser estimado em função da área foliar e da evaporação do tanque reduzido.

2. O consumo de água pelo crisântemo pode ser estimado em função da área foliar e da evaporação do atmômetro com escala de leitura ampliada.

\section{REFERÊNCIAS BIBLIOGRÁFICAS}

ALTHENHOFEN, J. A modified atmometer for on farm evapotranspiration determination. In: CONFERENCE ON ADVANCES IN EVAPOTRANSPIRATION, Chicago, 1985. Proceedings. Chicago: ASAE, 1985. p.177-184.

KAMPF, A.N. Floricultura: um negócio lucrativo. Trigo e Soja, v. 102, p.3-4, 1989.

KAMPF, E.; BAJAK, E.; JANK, M.S. O Brasil no mercado internacional de flores e plantas ornamentais. Informe GEP/DESR, v.3, n.4, p.3-11, abr. 1990.

MERCADO das flores. Informativo Semanal Cacex-Banco do Brasil, v. 22, n.1057, p.4-13, dez. 1987.

MONTERO, J.I.; CASTILLA, N.; GUTIERREZ de RAVÉ, E.; BRETONES, F. Climate under plastic in the Almeria. Acta Horticulturae, n.170, p.227-234, 1985.

ROSENBERG, N.J.; McKENNEY, M.S.; MARTIN, P. Evapotranspiration in a greenhouse-warmed world: A review and a simulation. Agricultural and Forest Meteorology. v. 47, p.303-320, 1989.

WILlMOTT, C.J. On the validation of models. Physical geography, v.2, n.2, p. 184-94, 1981. 